\title{
Example-based Chinese Calligraphy Synthesis
}

\author{
Wei $\mathrm{Li}^{1, *}$, Yumin $\mathrm{Chen}^{1}$, Chao Tang ${ }^{2}$ and Shaoyong $\mathrm{Yu}^{1}$ \\ ${ }^{1}$ School of Computer and Information Engineering, Xiamen University of Technology, China \\ ${ }^{2}$ Department of Computer Science and Technology, Hefei University, China \\ *Corresponding author
}

\begin{abstract}
Chinese calligraphy synthesis is important meaningful to protect non-heritage cultural and augmented reality. In this paper, we proposed an algorithm of Chinese Calligraphy synthesis based on examples. First, we construct a stroke library represented by vector. Second, we obtain the trajectory of character stroke from the digital tablet input, and match the most similar character stroke from the stroke library and replace the trajectory with it. Third, we evaluate the style using recurrent neural networks (RNNs). We also propose a new model to describe Chinese character topology by 9 -intersections \& range relation (9IRR) according to experience. Finally, we employ simulated annealing strategy to optimize the Chinese character topology with aesthetic requirements. Experimental results show that character shapes generated are visual pleasing.
\end{abstract}

Keywords- Chinese calligraphy synthesis; Chinese character topology; recurrent neural networks; optimization

\section{INTRODUCTION}

Chinese calligraphy art has a long history and is profound. So it is a very important non-cultural heritage. We can almost not get certain artist's Chinese calligraphy works. For example, there are only 324 characters in Lan Ting Xu of Xizhi Wang, who was a famous artist of Jin dynasty in ancient China. We may extend Xizhi Wang's works by computer. That is, we learn the style of Xizhi Wang calligraphy by algorithm and create more characters similar with Xizhi Wang's style. Thus, it is possible to reproduce Xizhi Wang's works. Calligraphy art and beauty have fascinated human beings from the emergence of text, inspiring countless artists and philosophers [1]. However, an absolute definition of aesthetic style remains difficult. For the complex of Chinese character topology, it is a challenge to generate a large-scale character set via a small-scale one. It is meaningful to construct Chinese character library.

In recent years, researchers proposed some synthesis methods on Chinese character. For example, Zhang Xiafen et al. developed computational tools for classification and quantification of calligraphic style and demonstrated on a statistically characterized corpus [2]. These works can be grouped into two classes roughly. One is automatic synthesizing and the other is interactive synthesizing. The main idea is to create a new style Chinese character by combing examples in automatic synthesis. In [3], the same Chinese characters with different style are input and created by deforming contour. Xu et al. [4] employ weighting different examples to generate new style Chinese character. $\mathrm{Xu}$ et al. [5] introduce complex inference to generate new style Chinese characters. In [6], they used character's skeleton to describe the character topology and generated a new style of character via controlling the skeleton's curve. Besides that, interactive methods are popular in synthesizing character. Dong et al. [7] simulate imaginal thinking and employ statistic model to generate Chinese calligraphy with new style.

Zhang et al. [8] replace trajectory with stroke and generate Chinese calligraphy. These methods rely heavily on the same character with different style to output characters with new style. In contrast, our methods are different with them. The similar style of different characters written by author is learned by computer. After that, we can convert the character synthesis problem to solving an optimization problem. That is, according to the learned style, we can obtain more characters with the similar style.

In addition, in visual reality field, some researches had been done about synthesis of style side. In [9], they empirically evaluated the effects of realistic vs. non-realistic stylized appearance of virtual humans on the emotional response of participants in a medical virtual reality system that was designed to educate users in recognizing the signs and symptoms of patient deterioration. Kim and his colleagues presented an adaptive data-driven algorithm for interactive crowd simulation [10].

In summary, the contributions of this paper are given as follows: 1) Firstly, a novel representation of Chinese character topology is proposed. 2) Secondly, RNNs is firstly used to evaluating Chinese calligraphy style. The rest of paper is organized as follows: Section 2 introduces the shape descriptor and recognition of trajectory. Section 3 provides 9IRR representing Chinese character topology. Learning style is introduced in Section 4 and Section 5. Optimizing characters via simulated annealing is provided in Section 6. Experiments are showed in Section 7. Conclusion is drawn in Section 8.

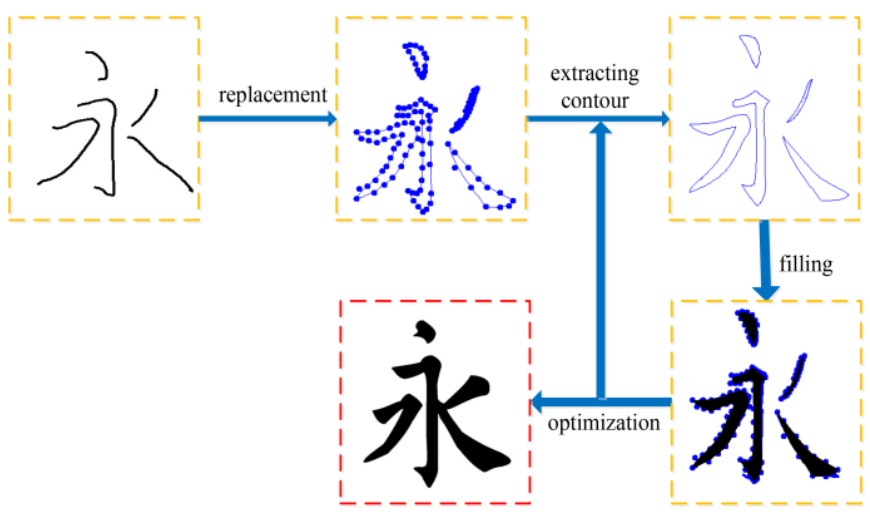

FIGURE I. THE OVERVIEW OF EXAMPLE-BASED CHINESE CALLIGRAPHY SYNTHESIS. 


\section{Chinese CAlligraphy TRA JECTORY RECOGNITION}

Chinese character is composed of the radical. Radical is combined by the stroke. The general strokes are shown in Fig. 1. Trajectory recognition requires matching objects with complicated trajectories. Shape context is used [11] because it describes the relative spatial distribution of land-mark points around feature points. For the shape context descriptor, two trajectories $\mathrm{A}$ and $\mathrm{B}$ are defined by the feature points as $\left\{A_{1}, A_{2}, \ldots, A_{n}\right\}$ and $\left\{B_{1}, B_{2}, \ldots, B_{n}\right\}$, respectively. The shape context of $A_{s}$ is defined as a histogram $h_{s}$ of the remaining $n-1$ points of the trajectory A under a global coordinate system: $h_{s}(c)=\#\left\{A_{j}: j \neq s, A_{j}-A_{s} \in \operatorname{bin}(c)\right\}$, where the bins uniformly divide the log-polar space. Similarly, $h_{t}(c)=$ $\#\left\{B_{j}: j \neq s, B_{j}-B_{s} \in \operatorname{bin}(c)\right\}$ is the shape context of $B_{t}$. The distance between the shape context of $A_{s}$ and $B_{t}$ is defined by [11] as

$$
D\left(A_{s}, B_{t}\right)=\frac{1}{2} \sum_{c=1}^{C} \frac{\left(h_{s}(c)-h_{t}(c)\right)^{2}}{h_{s}(c)+h_{t}(c)}
$$

The value of parameter $\mathrm{c}$ represents the number of bins of the histogram.

\section{CHINESE CHARACTER TOP OLOGY REPRESENTING}

Chinese character topology is defined as arrange and layout among strokes. We are inspired by [12] and propose a 9-
Intersections and Range Relation (9IRR) model to describe Chinese character topology. 9-Intersections are based on pointset topology theory. Symbol $\partial A$ represents the border of Object $\mathrm{A}$ and symbol $A^{0}$ represents the inner of $\mathrm{A}$. Symbol $A^{-}$ represents the exterior of A. 9-Intersection can distinguish 2 relationships between points, 3 relationships between point and line, 3 relationships between point and surface, 33 relationships between lines, 19 relationships between line and surface, 8 relationships between surfaces. 9-Intersections are completeness and simplicity in representing object topology. The topology between Object A and B can be defined as the follows.

$$
\text { Topology }(A, B)=\begin{array}{lll}
A^{0} \cap B^{0} & A^{0} \cap \partial B & A^{0} \cap B^{-} \\
\partial A \cap B^{0} & \partial A \cap \partial B & \partial A \cap B^{-}
\end{array}
$$

On the basis of equation (2), we add equation (3) as describing Chinese character topology. On the x-axis direction, it is as the follows. Similarly, on the y-axis direction, we can get equation (4) as the follows.

In which, $\operatorname{sgn}(\cdot)$ indicates sign function. Let Chinese character $C$ is composed of $C_{1}, C_{2}, \cdots, C_{n}$, where $C_{i}$ indicates $i t h$ stroke. Thus, according to equation (2)-(4), we can get 9IRR to describe $\mathrm{C}$, i.e. equation (5)-(7).

$$
\begin{aligned}
& \operatorname{RR}_{X}\left(C_{i}, C_{j}\right)=\left[\begin{array}{ll}
\operatorname{sgn}\left(\min \left\{x \mid x \in C_{i}\right\}-\min \left\{x \mid x \in C_{j}\right\}\right) & \operatorname{sgn}\left(\min \left\{x \mid x \in C_{i}\right\}-\max \left\{x \mid x \in C_{j}\right\}\right) \\
\operatorname{sgn}\left(\max \left\{x \mid x \in C_{i}\right\}-\min \left\{x \mid x \in C_{j}\right\}\right) & \operatorname{sgn}\left(\max \left\{x \mid x \in C_{i}\right\}-\max \left\{x \mid x \in C_{j}\right\}\right)
\end{array}\right] \\
& \operatorname{RR}_{Y}\left(C_{i}, C_{j}\right)=\left[\begin{array}{ll}
\operatorname{sgn}\left(\min \left\{y \mid y \in C_{i}\right\}-\min \left\{y \mid y \in C_{j}\right\}\right) & \operatorname{sgn}\left(\min \left\{y \mid y \in C_{i}\right\}-\max \left\{y \mid y \in C_{j}\right\}\right) \\
\operatorname{sgn}\left(\max \left\{y \mid y \in C_{i}\right\}-\min \left\{y \mid y \in C_{j}\right\}\right) & \operatorname{sgn}\left(\max \left\{y \mid y \in C_{i}\right\}-\max \left\{y \mid y \in C_{j}\right\}\right)
\end{array}\right]
\end{aligned}
$$

$\operatorname{TOPO}(\mathrm{C})=\left(\begin{array}{ccc}\operatorname{Topo}\left(\mathrm{C}_{1}, \mathrm{C}_{1}\right) & \cdots & \operatorname{Topo}\left(\mathrm{C}_{1}, \mathrm{C}_{\mathrm{n}}\right) \\ \vdots & \ddots & \vdots \\ \operatorname{Topo}\left(\mathrm{C}_{\mathrm{n}}, \mathrm{C}_{1}\right) & \cdots & \operatorname{Topo}\left(\mathrm{C}_{\mathrm{n}}, \mathrm{C}_{\mathrm{n}}\right)\end{array}\right)$

RangeRelation $_{X}(C)\left(\begin{array}{ccc}\operatorname{RR}_{X}\left(C_{1}, C_{1}\right) & \cdots & R_{X}\left(C_{1}, C_{n}\right) \\ \vdots & \ddots & \vdots \\ \operatorname{RR}_{X}\left(C_{n}, C_{1}\right) & \cdots & R_{X}\left(C_{n}, C_{n}\right)\end{array}\right)$

RangeRelation $_{Y}(C)=\left(\begin{array}{ccc}\operatorname{RR}_{\mathrm{Y}}\left(\mathrm{C}_{1}, \mathrm{C}_{1}\right) & \cdots & \mathrm{RR}_{\mathrm{Y}}\left(\mathrm{C}_{1}, \mathrm{C}_{\mathrm{n}}\right) \\ \vdots & \ddots & \vdots \\ \operatorname{RR}_{\mathrm{Y}}\left(\mathrm{C}_{\mathrm{n}}, \mathrm{C}_{1}\right) & \cdots & \mathrm{RR}_{\mathrm{Y}}\left(\mathrm{C}_{\mathrm{n}}, \mathrm{C}_{\mathrm{n}}\right)\end{array}\right)$

\section{RECURRENT NEURAL NETWORKS}

The recurrent neural networks connect the nodes of the hidden layer, and the input of the hidden layer is not only the output of the input layer at the current moment but also the output of the hidden layer at the last moment. In this way, the neural networks can be used to remember information from the current moment in the network [13]. The structure of the recurrent neural networks are roughly the same as that of the feed-forward neural network, and the difference lies in the connection between the hidden layer, which increases the weight between the hidden layers $W_{h}$. If the hidden layer $L_{\text {hidden }}$ includes $m$ neurons, $W_{h}$ is $\mathrm{m} \times \mathrm{m}$ weight matrix. The propagation process of recurrent neural networks can be expressed in equation (2):

$$
\begin{aligned}
& h_{t}=f\left(W_{1}^{T} x+W_{h}^{T} h_{t-1} x+b_{1}\right) \\
& y=f\left(W_{2}^{T} h_{t}+b_{2}\right)
\end{aligned}
$$

where $W_{1}$ represents the connection weight between the input layer and the hidden layer, $W_{2}$ denotes the connection weight between the hidden layer and the output layer, $b_{1}$ and $b_{2}$ respectively represent the bias of the hidden layer and the output layer, $x_{t}, h_{t}$ and $y_{t}$ respectively represent the input, the output of the hidden layer and the output of the network at time $t . h_{t-1}$ is the output of the hidden layer at time $t-1$.

\section{LEARNING STYLE}

In order to reduce the number of sample, we compute the distance between the Chinese character with standard style and the one with special style as features. We invite artist to give scores for the samples. Thus, we can get the labeled samples. Let $X$ be a Chinese character with special style and $\hat{X}$ be a standard style. We can compute the distance between $X$ and $\hat{X}$ on 9IRR. 


$$
\begin{gathered}
D_{1}(X, \hat{X})=\operatorname{TOPO}(X)-\operatorname{TOPO}(\hat{X}) \\
D_{2}(X, \hat{X})=\text { RangeRelation }_{x}(X)-\text { RangeRelation }_{x}(\hat{X}) \\
D_{3}(X, \hat{X})=\text { RangeRelation }_{y}(X)-\text { RangeRelation }_{y}(\hat{X})
\end{gathered}
$$

After that, we compute matrix $D_{i}$ and $A_{i}=D_{i} * D_{i}^{T}, i=$ $1,2,3$. We compute the maximum, minimum, maximum absolute, mean, median and maximum eigenvalue for $A_{i}$. So we can get 18-dimensional feature vector and evaluation score (on a scale of 1-5). These are used as training samples. We employ RNNs to train the samples. RNNs is artificial neural network with inner neural element connected each other. The connection let the inner of RNNs be with complex dynamic properties.

\section{OPTIMIZATION}

We are inspired by [1] and employ the simulated annealing algorithm as optimization of Chinese character. However, we modify cost function on the basis of [1], i.e., we add the history evaluation factor to the cost function. The cost function is as the follows.

$$
\begin{gathered}
E(X)=\omega_{1} * \text { Evaluate }(X)+\omega_{2} * \operatorname{Depth}(X)+\omega_{3} * \\
\text { History }(X) \\
(12)
\end{gathered}
$$

Where Evaluate $(\cdot)$,Depth $(\cdot)$,History $(\cdot)$ indicate the current evaluation score, the depth of tree and the last evaluation score respectively.

We define an affine transformation $T_{i}$ including rotation, translation, scaling. Let a Chinese character $C$ be composed of $C_{j}, j=1,2,3, \ldots, N$. In each iteration procedure, generating two random represent affine transformation index and stroke index respectively. Thus, we can get a new style of character after transformation $T_{i, j}^{k}(C)$ where represents $i t h$ transformation of the stroke $C_{j}$ in the $k t h$ iteration. After computing $E\left(T_{i, j}^{k}(C)\right)$, we compare it with $E\left(T_{i_{1} j_{1}}^{k-1}\right)$. If $E\left(T_{i, j}^{k}(C)\right)<E\left(T_{i_{1} j_{1}}^{k-1}\right)$, we accept the change. Otherwise, we accept the change as probability $p=\exp (-\delta / T(t)) . \delta$ is the difference of energy and $T(t)=T_{0} / \lg (1+t) . T_{0}$ is const and $t$ represents $t$-th iteration.

\section{EXPERIMENTAL RESULTS AND DISCUSSIONS}

We demonstrate and discuss results obtained by our algorithm in this section. FIGURE II shows the optimization process of the Chinese character "big" and "middle". We can find the results are much better than the original input. FIGURE III shows a Chinese idiom and it means that we can learn something new by reviewing what has been learned. As shown in FIGURE III, we write the trajectory in the upper row and get the optimization results in the second row. Experiments demonstrate the recognition rate is over $97 \%$ using shape context algorithm. It costs average 1.01 seconds in generating a character. We also get Chinese calligraphy with different style via corresponding strokes as shown in FIGURE IV. These characters are composed of strokes from Xizhi Wang's works. In FIGURE V, we synthesize Chinese calligraphy with Gongquan
Liu's style. In FIGURE V (a), there are some handwritten characters and they were replaced by strokes from Gongquan Liu's works. After optimization, we have synthesis works in FIGURE V (b). As shown in FIGURE VI, using [1], [14] and our method, we employ official script strokes to synthesize some works. In FIGURE VI (a), we can see synechia among strokes is a little heavy via [1], especially FIGURE VI (a-1). In FIGURE VI (b), we find Chinese character's topology is a little compact. As shown in FIGURE VI (c), these works are more similar with official script style using our method. Also, as TABLE I shown, during synthesis, our method is more excellent than the other two methods mentioned, such as evaluation score, synthesis time, optimization time, trace recognition.

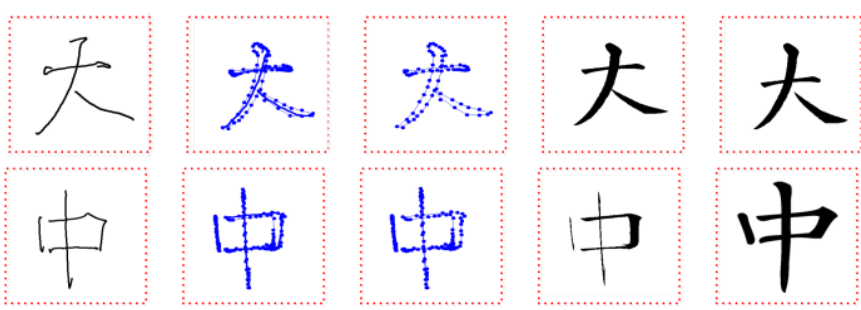

FIGURE II. THE GENERATION PROCESS OF THE CHINESE CHARACTER BIG AND MIDDLE.

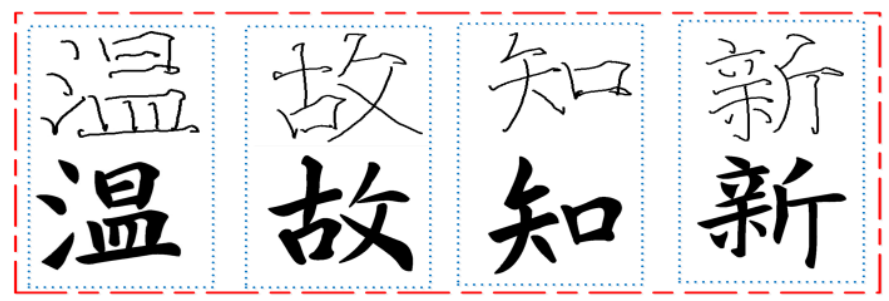

FIGURE III. CHINESE IDIOM

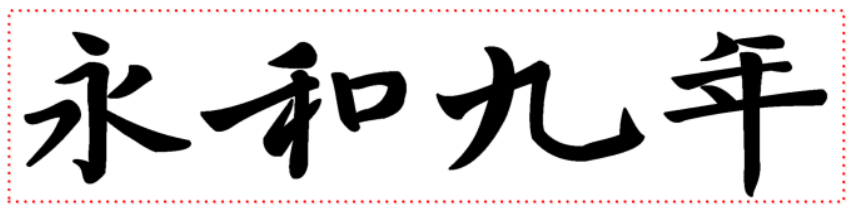

FIGURE IV. THE CHINESE CALLIGRAPHY WITH XIZHI WANG'S STYLE

TABLE I. COMPARISON WITH PARAMETERS DURING SYNTHESIZING WORKS.

\begin{tabular}{|l|c|c|c|c|}
\hline Method & $\begin{array}{c}\text { Score } \\
\text { (average) }\end{array}$ & $\begin{array}{c}\text { Trace } \\
\text { Recognition }\end{array}$ & $\begin{array}{c}\text { Synthesis } \\
\text { Time } \\
\text { (average) }\end{array}$ & $\begin{array}{c}\text { Optimization } \\
\text { Times } \\
\text { (average) }\end{array}$ \\
\hline$[1]$ & 4.2 & $93 \%$ & $1.13^{\prime}$ & 600 millseconds \\
\hline$[14]$ & 4.5 & $94 \%$ & $1.05^{\prime}$ & 643 millseconds \\
\hline Ours & 4.7 & $97 \%$ & $1.01^{\prime}$ & 620 millseconds \\
\hline
\end{tabular}

\section{CONCLUSION}

In this paper we introduced 9IRR to represent the Chinese character's topology. And we employ RNNs as learning and evaluating of Chinese calligraphy. In the character's optimization process, we introduced simulated annealing algorithm. This is an exploration into the challenging task of generating large-scale glyph sets with small-scale ones. In addition, 'hollow-strokes' effect is very important style and we consider more factors in future. 

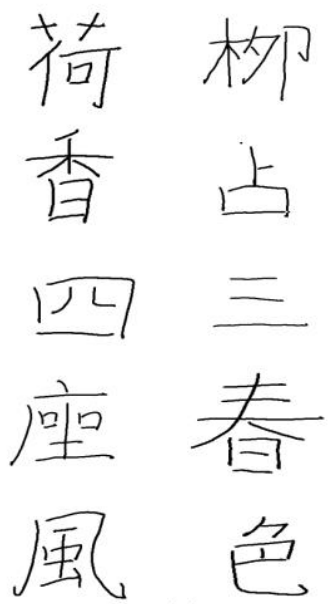

(a)

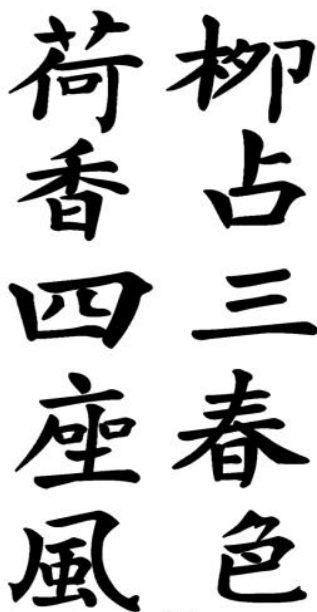

(b)

FIGURE V. THE CHINESE CALLIGRAPHY WITH GONGQUAN LIU STYLE.

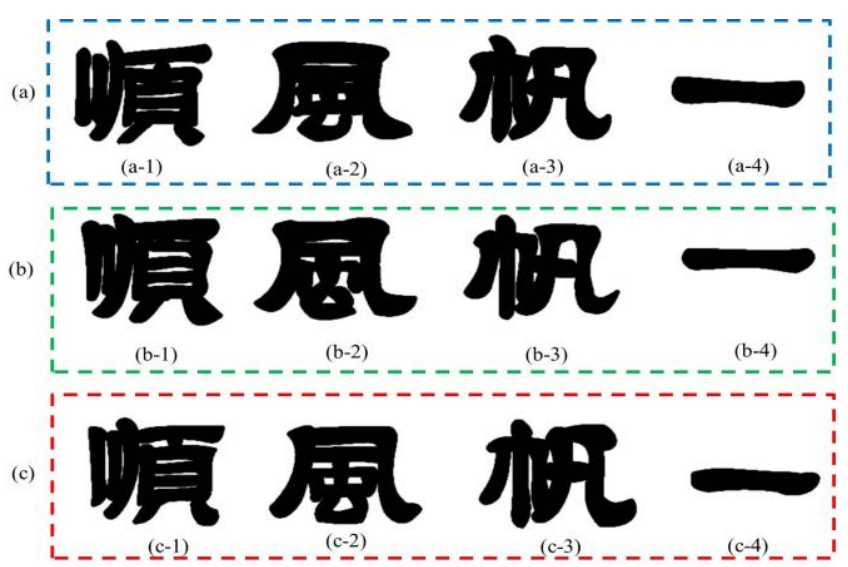

FIGURE VI. CHINESE CALLIGRAPHY WITH OFFICIAL SCRIPT STYLE USING DIFFERENT METHODS.

\section{ACKNOWLEDGEMENTS}

This work was supported by Natural Science Foundation of Fujian province of China (No. 2016J01325 and No. 2015J05015), Scholarship under the Education Department of Fujian Province as a visiting scholar, Guidance Project of Science and Technology of Xiamen (No. 3502Z20179038), Natural Science Foundation of China (No. 61573297), Highlevel Talents Foundation of Xiamen University of Technology (No. YKJ14014R), Scientific Research Fund Project of Talents of Hefei University (No. 15RC07), Natural Science Research Project of Universities of Anhui Province (No. KJ2015A206), Open Project Foundation of Intelligent Information Processing Key Laboratory of Shanxi Province (No. 2014001), Excellent Talents Training Funded Project of Universities of Anhui Province (No. gxfx2017099).

\section{REFERENCES}

[1] Wei Li and Changle Zhou, "Data-driven Enhancement of Chinese Calligraphy Aesthetic Style," Journal of information and Computional Science, vol. 10, pp. 3645-3658, 2013.

[2] Xiafen Zhang and George Nagy. "Computational method for calligraphic style representation and classification," Journal of Electronic Imaging, vol. 24 , no. 5 , pp. $1-9,2015$
[3] Xiafen Zhang and Guangzhong Liu, "Chinese calligraphy character image synthesis based on retrieval," Lecture Notes in Computer Science, vol. 5879, pp. 167-178, 2009.

[4] Songhua Xu and Hao Jiang and Tao Jin and Francis C.M Lau and Yunhe Pan, "Automatic generation of Chinese calligraphic writings with style imitation," IEEE Intelligent Systems, vol. 24, pp. 44-53, 2009.

[5] Songhua Xu and Francis C.M Lau and William K Cheung and Yunhe Pan, "Automatic generation of artistic Chinese calligraphy," IEEE Intelligent Systems, vol. 20, pp. 32-39, 2005.

[6] Rapee Suveeranont and Takeo Igarashi, "Example-based automatic font generation," SMART GRAPHICS Lecture Notes in Computer Science, vol. 6133, pp. 127-138, 2010.

[7] Jun Dong and Miao Xu and Xianjun Zhang and Yanqing Gao and Yunhe Pan, "The creation process of Chinese calligraphy and emulation of imagery thinking," IEEE Intelligent Systems, vol. 23, pp. 56-62, 2008.

[8] Zhenting Zhang and Jiagnqin $\mathrm{Wu}$ and Kai Yu, "Chinese calligraphy specific style rendering system, " Proceedings of the ACM International Conference on Digital Libraries, pp. 99-108, 2010.

[9] Volante M. and Babu SV and etc. "Effects of virtual human appearance fidelity on emotion contagion in affective inter-personal simulations," IEEE Trans Vis Comput Graph, vol. 22, no. 4, pp.1326-1335, 2016.

[10] Sujeong Kim and Aniket Bera and etc. "Interactive and adaptive datadriven crowd simulation," Virtual Reality (VR), pp. 19-23, July 2016.

[11] Serge Belongie and Jitendra Malik and Jan Puzicha, "Shaping Matching and Object Recognition Using Shape Contexts," IEEE Transactions on Pattern Analysis and Machine Intelligence, vol. 24, pp. 509-522, 2002.

[12] Zhanlong Chen, Qiqi Feng and Xincai Wu, "Represention Model of Topological Relations between Complex Planar Objects," Acta Geodaetica et Cartographica Sinica, vol. 44, pp. 438-452, 2015.

[13] E.B. Kosmatopoulos, M.M. Polycarpou, M.A. Christodoulou, P.A. Ioannou. "High-order neural network structures foridentification of dynamical systems," IEEE transactionson neural networks, vol. 6, no. 2, pp. 422-431, 1995.

[14] Wei Li and Changle Zhou and Yuping Song, "Computationally evaluating and synthesizing Chinese calligraphy," Neurocomputing, vol. 135, pp. 299-305, 2014. 